\title{
Exterior design of Southwest hills multifunctional mini-chassis
}

\author{
Haochen Han $^{1, ~ a}$, Yongliang Wen ${ }^{1, b}$, Tingyu Wang ${ }^{1, c}$, and Xiaorong LV $^{1, d^{*}}$ \\ 1College of mechanical and electrical engineering, Sichuan Agricultural University, Ya'an, 625014, \\ China \\ aemail:872426951@qq.com, email:1696793379@qq.com, cemail:964026857@qq.com,
}

Keywords: multifunctional mini-chassis, exterior design, 3D modeling

Abstract. There are the chassis exposed and the lack of cockpit problems in Southwest hills multifunctional mini-chassis. In the case of meeting practice, this exterior design has simple lines and generous model. The unique detachable box has solved the heat radiating, drainage and other key issues, and users are satisfied with the fully enclosed cockpit in farmland. Through the virtual prototype technology, we have completed the whole 3D modeling.

\section{Introduction}

Southwest hills are the main producing areas of food crops, but because of geographical conditions, cropping systems and plant size and other factors, production power are still manpower and animal power. Multifunctional mini-chassis has its own characteristics, especially for southwest hills intercropping planting pattern's need [1] .At present, the lack of practical's chassis appearance will affect machine performance and it is difficult to give a test or a universal. In general, agricultural machinery is measured by the technical and artistic appearance of two indicators. The technical beauty of the appearance mainly means that the design of the tractor cab is consistent with people operational requirements and ensures the process of driving comfortably and safely in order to meet the requirements high efficiency and accuracy. The art of sculpture emphasizes the amenity of sculpture, appearance quality and the pleasant and delicate products will give users a great visual experience [2].

The design of southwest hills multifunctional mini-chassis combines with aesthetic principles, body composition, ergonomics and other theory and it has planned and integrated lines of appearance, color and decoration. There are removable structure, good heat dissipation, cabinet and delicate look in the design of power box. In the cockpit side, fully enclosed design meets the operational requirements for the driver.

\section{Structure and Design idea}

\section{The overall structure of Appearance design}

The appearance of southwest hills multifunctional mini-chassis "Chuannong LZ-1" consists of the cockpit, power box and chassis three major components. The 3D model of appearance design is shown in Fig.1.

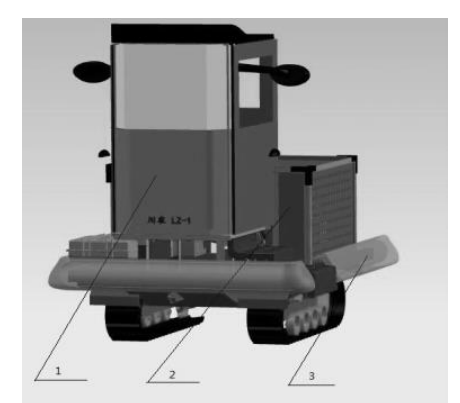

Fig.1 multifunctional mini-chassis 3D model of appearance

1 cockpit; 2 power box; 3 chassis 


\section{The design ideas of Appearance design}

In this paper, because the body of the appearance design belongs to small machine, it must take into account the security, economic, practice, beauty and other factors. The design with simple lines is the core, and the power box with the angle steel and thin steel plate creates a generous and beautiful overall style. The chassis box emphasizes the package and the beauty of chassis. Fully enclosed cockpit meets the requirement of users in the fields of vision, anti-skid, anti-noise, damping of vibration, drainage. Through installation, practice and beauty three aspects, the design can increase the added value of product.

\section{The main structural design}

In this paper, the exterior design of southwest hills multifunctional mini-chassis is design through the virtual 3D. Virtual design technology provides a product design environment, optimize the product design process, to research and development of new products provides a strong support. By using the virtual design technology, which can improve the design quality, reduce design errors and manufacturing costs. The design of southwest hills multifunctional mini-chassis exterior is designed by UGNX9.0 software [3].

\section{Cockpit structural design}

The cockpit which used the design idea of fully enclosed structure, mainly include: bridge, ceiling, door, front and back windows and rearview mirrors. The 3D model of cockpit is shown in Fig. 2.

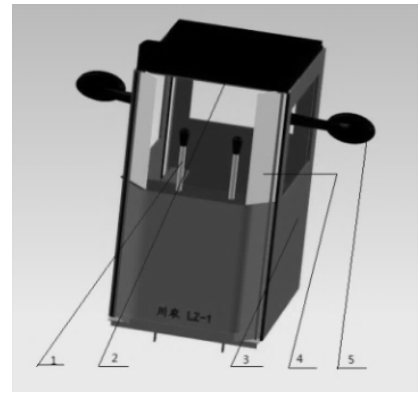

Fig. 2 the 3D model of cockpit

1 bridge; 2 ceiling; 3door; 4 front and back windows; 5 rearview mirrors

Driving station uses the lever to control the vehicle forward direction and by changing two-track speed to achieve the purpose of turning; and the size of the curve track is by speed difference and the distance of difference. By operating rod with non-slip pattern, you can spend less effort operating it. Circular groove can bloom water cup. The designed armrest next to the seat is convenient for drivers to rest.

The ceiling uses the symmetrical tilting style's shape. There are many rainy days in southwest region, and the tilting design can avoid water to influence front window's vision, tilting left and right borders are in favor of drainage. Fluid sense of the overall shape can give people fluent and comfortable feeling.

Left and right door frame installed glass. Door switch is oval pie, and its appearance is derived from the panda's tail. The front windows are divided into two big ones and a small one, and the two sides are made into oblique as backdrop. The back door is similar to the left and right doors. The glass can provide adequate vision when driving the tractor, and reduce the risk of driving danger.

Panda ear type's rearview mirrors. On the one hand, they can help the driver to drive safely and people can easily observe the back view. On the other hand, the smooth, rounded shape, compared with ordinary, square and neatly mirrors, is less rigid and boring, and greatly enriches people's visual experience.

Fully enclosed cockpit can play a very good sound and heat insulation effect and provide clean, good working environment. At the same time, it can guarantee a good driver's personal safety. 


\section{Power box structural design}

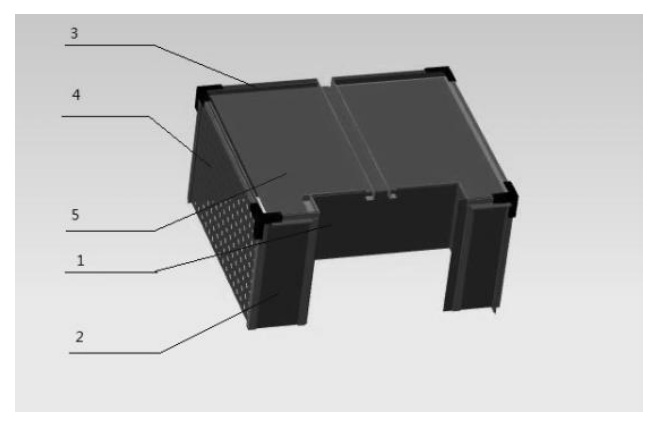

Fig. 3 the 3D model of power box

1 front main baffle;2 front side baffle;3 behind baffle;4 left and right baffle;5 upper baffle

Power box mainly includes: front main baffle, front side baffle, behind baffle, left and right baffle and upper baffle. The box is divided into two symmetrical parts, and each part connected with bolts. There are two small baffles can be opened in behind baffle. It can be used when the machine is break down. The appearance of the skeleton is smooth and fluent, and the entire structure gives a sense of compact feeling. On the other hand, each steel plate is self-contained and is easily to repair and dismantle. The 3D model of power box is shown in Fig.3.

Entire frame's quality is very light, the slab uses 3mm's thin steel and will not cause too much impact on the tractor load. The left and right steel plate of the diamond is convenient to radiate when the unit is working.

\section{Chassis box structural design}

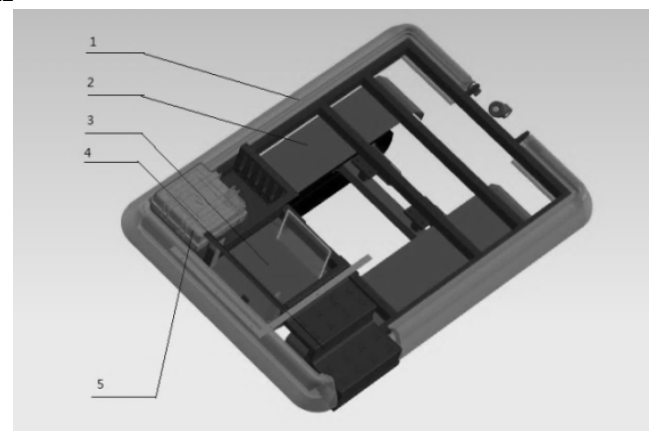

Fig. 4 the 3D model of chassis box

1 chassis barrier; 2 Fender; 3 fault traction device; 4 the ladder; 5 toolbox

The chassis body mainly has chassis, guard rails, fender, the fault traction device, the ladder and toolbox. The 3D model of chassis box is shown in Fig.4.

Chassis barrier adopt irregular smooth semi-circular appearance. Small tracked chassis can find a match with a fine shape. Track mud tile can prevent mud polluting engine chassis. Fault traction device is similar to traction ring. Ladders, in view of a small tractor's design, according to the people's habit of up and down stairs, are designed for two. And a non-slip surface design in ladder lines can reduce the risk of slipping when upstairs and downstairs. On the box fence's left side, we designed a toolbox for holding tools, parts.

\section{Appearance design features}

The design of this paper in art, power box has the style of flat surface, cockpit has the style of angular surface and chassis box has the style of curved surface. All those are to shape the stability of tractor. People will feel perfect when unified and orderly flat surface style, fluent angular surface style and round surface style are combined. In safe and practical aspects, enclosed cockpit play a role in sound and heat insulations for the driver. In other word, it also provides a clean fine driving environment. The angle steel skeleton in cockpit and power box both can meet the requirements of the manufacture, 
installation and repair aspects. It is convenient for people to check and repair machine in daily life if we have a toolbox. Fault traction device can well solve the problem of the delivery of the trouble machine.

\section{Mechanism color configuration}

The main color of the body is dark green. Auxiliary color is black and red. Power box, Chassis box and Cockpit choose the green with a sense of progress and expansion, in order to have a warning effect in the chaos construction environment. The cab roof, angle steel, track and chimney choose black. Track wheel chooses red to increase the highlights of the appearance.

\section{Conclusion}

The exterior design of southwest hills multifunctional mini-chassis exterior takes detachable box and enclosed cockpit as core. The overall design has the advantages of safe and comfortable use, easy to be manufactured and convenient disassembly, etc. In field operations, it can well reduce the labor intensity of the driver and improve working efficiency. So it can achieve the design requirements of inexpensive. In a word, the design can play a certain role in the popularization of the multifunctional mini-chassis in the southwest.

\section{Acknowledgements}

The study was supported by the National Natural Science Foundation of China (51105261)

Correspondence Author: Lv Xiaorong, College of mechanical and electrical engineering, Sichuan Agricultural University, Ya’an, China, lxrxj2008@163.com.

\section{References}

[1] Xiaorong Lv, Weimin Ding. Agricultural Mechanization Research, No.9 (2013), P.250-252, In Chinese

[2] Li Xin, Jinggang Yi, Jiangtao Liu, Haiyong Jiang, Wenhui Hou. Agricultural Mechanization Research, No.5 (2008), P.210-212, In Chinese

[3] Lihua Zhang, Xiaorong Lv. UG NX9.0 computer-aided design and making practical tutorial. Beijing: Peking University Press. (2015), In Chinese 